\title{
Keratoacanthoma and squamous cell carcinoma are distinct from a molecular perspective
}

Seong $\mathrm{H} \mathrm{Ra}^{1,2}$, Albert $\mathrm{Su}^{2}$, Xinmin $\mathrm{Li}^{2}$, Jaime Zhou ${ }^{2}$, Alistair J Cochran ${ }^{2}$, Rajan P Kulkarni ${ }^{3}$ and Scott W Binder ${ }^{2}$

${ }^{1}$ San Diego Pathology Medical Group, San Diego, CA, USA; ${ }^{2}$ Department of Pathology and Laboratory Medicine, David Geffen School of Medicine at UCLA, Los Angeles, CA, USA and ${ }^{3}$ Department of

Dermatology, David Geffen School of Medicine at UCLA, Los Angeles, CA, USA

\begin{abstract}
Keratoacanthoma is a controversial entity. Some consider keratoacanthoma as a variant of squamous cell carcinoma, whereas others see it as a distinct self-resolving squamoproliferative lesion. Our objective is to examine the relationship of keratoacanthoma with squamous cell carcinoma and normal skin by using DNA microarrays. DNA microarray studies were performed on formalin-fixed and paraffin-embedded blocks from ten cases of actinic keratoacanthoma utilizing the U133plus2.0 array. These results were compared with our previously developed microarray database of ten squamous cell carcinoma and ten normal skin samples. Keratoacanthoma demonstrated 1449 differentially expressed genes in comparison with squamous cell carcinoma ( $>$ 5-fold change: $\boldsymbol{P}<0.01$ ) with 908 genes upregulated and 541 genes downregulated. Keratoacanthoma showed 2435 differentially expressed genes in comparison with normal skin ( $>5$-fold change: $P<0.01$ ) with 1085 genes upregulated and 1350 genes downregulated. The most upregulated genes, comparing keratoacanthoma with normal skin included MALAT1, S100A8, CDR1, TPM4, and CALM1. The most downregulated genes included SCGB2A2, DCD, THRSP, ADIPOQ, adiponectin, and ADH1B. The molecular biological pathway analysis comparing keratoacanthoma with normal skin showed that cellular development, cellular growth and proliferation, cell death/apoptosis, and cell cycle pathways are prominently involved in the pathogenesis of keratoacanthoma. The most enriched canonical pathways were clathrin-mediated endocytosis signaling, molecular mechanisms of cancer and integrin signaling. The distinctive gene expression profile of keratoacanthoma reveals that it is molecularly distinct from squamous cell carcinoma. The molecular pathways and genes differentially expressed in comparing keratoacanthoma with normal skin suggest that keratoacanthoma is a neoplasm that can regress due to upregulation of the cell death/apoptosis pathway. Modern Pathology (2015) 28, 799-806; doi:10.1038/modpathol.2015.5; published online 13 February 2015
\end{abstract}

Keratoacanthoma is a crateriform squamous lesion of the sun-exposed skin of elderly patients that typically grows rapidly then involutes. Microscopically, keratoacanthoma shows three distinctive growth phases including: the early growth phase; fully developed/proliferative phase; and senescent/ regressive phase. ${ }^{1}$ Most keratoacanthoma are biopsied in the fully developed/proliferative phase which is histologically characterized by a symmetric crateriform, exo-endophytic lesion with buttress formation and a prominent central keratin

Correspondence: Dr SH Ra MD, San Diego Pathology, 7592 Metropolitan Drive, Suite 405/406, San Diego, CA 92108, USA.

E-mail: sra@mednet.ucla.edu

Received 14 October 2014; revised 6 December 2014; accepted 9

December 2014; published online 13 February 2015 plug. Invasive lobules and nests of tumor cells with low-grade nuclei and abundant, glassy eosinophilic cytoplasm that mature toward the center are characteristic. Keratoacanthoma usually shows a sharp delineation between the tumor nests and stroma and can entrap elastic fibers. A prominent associated mixed inflammatory infiltrate of lymphocytes, eosinophils, and neutrophils is frequent. ${ }^{1,2}$

Keratoacanthoma remains controversial. Some believe that it is a variant of squamous cell carcinoma because of rare reports of metastasis. ${ }^{3,4}$ Others believe that keratoacanthoma is a distinct selfresolving benign squamoproliferative lesion., ${ }^{1,2,5-7}$ To investigate the relationship of keratoacanthoma to squamous cell carcinoma and normal skin, we analyzed the gene expression profile of over 47000 
genes using one of the most comprehensive GeneChip microarrays available (human U133 plus 2.0 array) to study differential gene expression between keratoacanthoma and squamous cell carcinoma and keratoacanthoma and normal skin in formalin-fixed and paraffin-embedded tissue.

\section{Materials and methods}

\section{Sample Selection}

Ten cases of actinic keratoacanthoma in the fully developed/proliferative stage (Figure 1) were identified from the Tamtron database. Slides and formalin-fixed and paraffin-embedded tissue $(<1$ since excision) were retrieved. The histology was

a

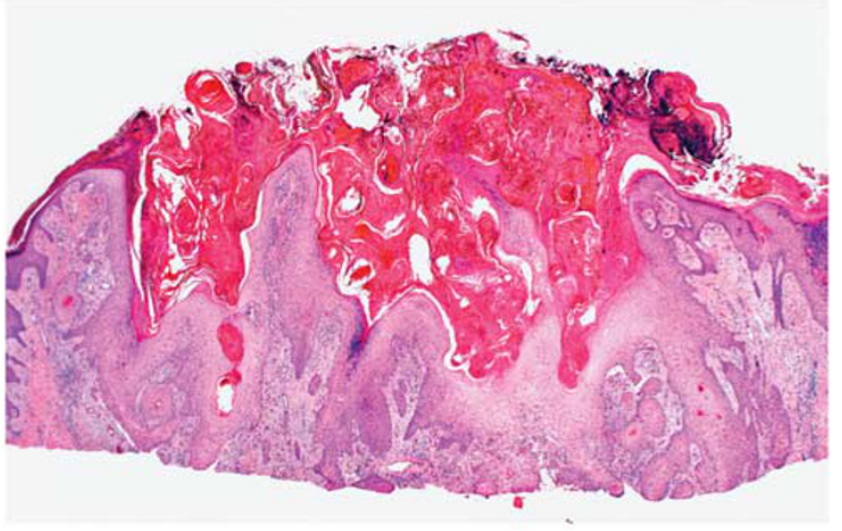

b

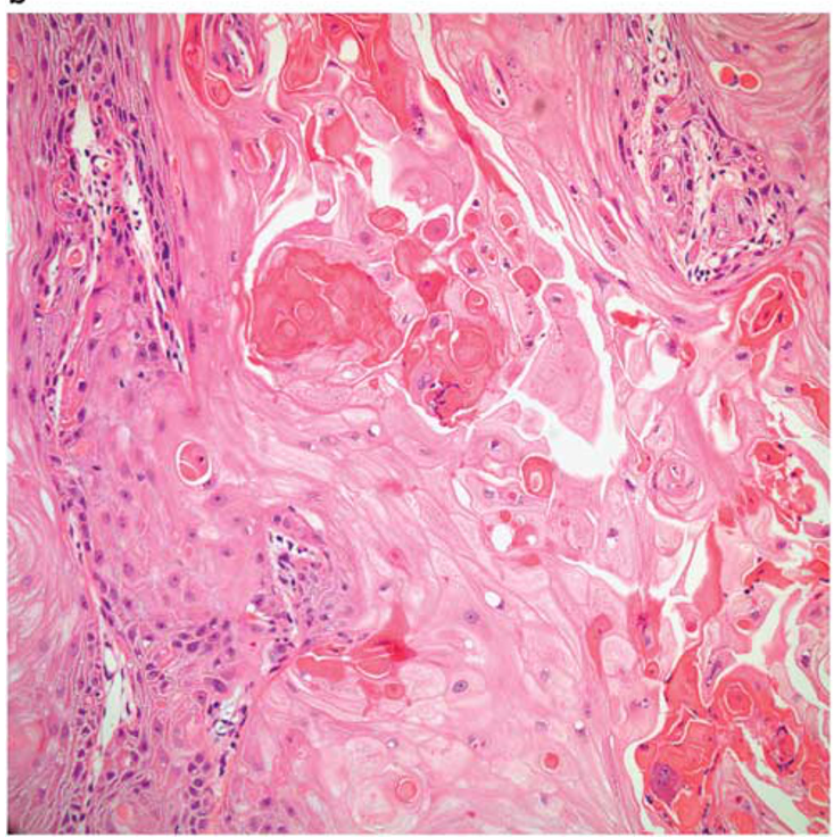

Figure 1 (a) Keratoacanthoma with crateriform architecture, central hyperkeratotic plug, and surrounding buttress of squamous epithelium (H\&E 20X). (b) Keratoacanthoma showing proliferating squamoid cells with abundant pale eosinophilic cytoplasm (H\&E 200X). reviewed and their diagnoses coincided. None of these tumors recurred or metastasized. Areas of interest were removed from the paraffin blocks with a sterile surgical scalpel. The ten cases of squamous cell carcinoma that were used for the comparison were previously reported. ${ }^{8,9}$ They consisted of well-to-moderately differentiated squamous cell carcinoma arising from skin showing actinic damage with solar elastosis and/or actinic keratosis. The ten cases of keratoacanthoma included sporadic keratoacanthomas and BRAF inhibitor-induced keratoacanthomas. Previous studies demonstrated no induction of MAP kinase pathway expression or significant molecular differences between keratoacanthomas from patients on BRAF inhibitors and patients not on inhibitors. ${ }^{10}$ Methods of RNA isolation, quality control, target preparation and microarray hybridization, and data analysis are described below and in our previous studies. ${ }^{8,9}$

\section{RNA Isolation and Quality Control}

Total RNA was isolated using the Ambion RecoverAll (Applied Bio systems/Ambion, Austin, TX, USA) kit according to the manufacturer's instructions. Briefly, formalin-fixed and paraffin-embedded samples were deparaffinized by using a series of xylene and ethanol washes and then subjected to a proteinase $\mathrm{K}$ digestion at $50{ }^{\circ} \mathrm{C}$ for $16 \mathrm{~h}$ to release RNA from covalently linked proteins. Finally, total RNA was purified by capturing on a glass-fiber filter. After washing, the total RNA was eluted. RNA integrity was evaluated by using an Agilent 2100 Bio analyzer (Agilent Technologies, Palo Alto, CA, USA) and purity/concentration was determined by using a NanoDrop 8000 (NanoDrop Products, Wilmington, DE, USA). The RNA samples with RNA integrity number $\geq 5$ and $260 / 280$ ratio $\geq 1.7$ were selected for the microarray.

\section{Target Preparation and Microarray Hybridization}

Microarray targets were prepared by using the NuGEN WT-Ovation formalin-fixed and paraffinembedded RNA Amplification System V2. This system offers the most efficient cDNA amplification powered by Ribo-SPIA technology and is ideal for global gene expression analysis with the small amount of degraded RNA derived from formalinfixed and paraffin-embedded samples. Fifty nanograms of the total RNA were used for the first-strand synthesis. After the second-strand cDNA synthesis, the double-stranded cDNA was purified using Agencourt RNAClean beads provided with the WTOvation kit, followed by SPIA cDNA Amplification. Five micrograms of amplified cDNA were fragmented and labeled by using NuGEN's FL-Ovation cDNA Biotin Module V2 according to the instructions (NuGEN Technologies, San Carlos, CA, USA) and then hybridized to the Affymetrix GeneChip U133 
plus 2.0 array (Affymetrix, Santa Clara, CA, USA) according to the manufacturers' instructions. The arrays were washed and stained with streptavidin phycoerythrin in Affymetrix Fluidics Station 450 by using the Affymetrix GeneChip protocol and scanned by using an Affymetrix GeneChip Scanner 3000.

\section{Data Analysis}

The acquisition and initial quantification of array images were conducted by using AGCC software (Affymetrix). The subsequent data analyses were performed using Partek Genomics Suite Version 6.6 (Partek, St Louis, MO). We first performed an ANOVA analysis to identify genes between the groups at $P<0.05$ and then calculated relative difference in fold change between the groups. Genes expressed at $\geq 2$-fold and $P<0.05$ were considered as differentially expressed between the groups. Cluster analyses were conducted with Partek default settings. Canonical pathway analyses were performed using the Ingenuity Pathway Analysis Version (Ingenuity Systems, Redwood City, CA, USA). Briefly, a differentially expressed gene list containing gene identifiers and corresponding fold changes was first uploaded as an Excel spreadsheet into the software. Each gene identifier was mapped to its corresponding gene object in the Ingenuity Pathways Knowledge Base. These genes were then used as the starting point for the pathway analysis. Canonical pathway analysis identified the pathways from the Ingenuity Pathways Analysis library of canonical pathways that were most significant to the data set. The significance of the association between data set and canonical pathway was measured in two ways: (1) a ratio of the number of genes from the data set that map to the pathway divided by the total number of genes that map to the canonical pathway was displayed; and (2) a Fischer exact test was used to calculate a $P$-value determining the probability that the association between the genes in the data set and the canonical pathway is explained by chance alone.

\section{Quantitative Real-Time PCR Analyses}

After analyzing our DNA microarray data of differentially expressed genes between keratoacanthoma and squamous cell carcinoma, three representative upregulated genes (CDR1, MALAT1, and NCRNA00084) and two representative downregulated genes (LOC441461 and CEP170) were selected for QRT-PCR confirmation. The QRT-PCR confirmation was performed by using the SYBR Green real-time RT-PCR kit (Applied Biosystems) according to the manufacturer's instructions. An additional three cases each of keratoacanthoma and squamous cell carcinoma were selected for the analysis and RNA isolation was performed by using the same protocol as above. The Applied Biosystems 7500
Real-Time PCR System was used for the analyses with the following primers:

CDR1: forward TTTGATGGAAGACCTTGAAATT AT; reverse TGTCTTCCAAGAAGCTCCAAG MALAT1: forward CCAAAAGAGAACCACACAC TACC; reverse GGTTGGTCTGGCCTACTGGG NCRNA00084: forward TCACTTGAAAGATGGGC GTCA; reverse ACCTAAGTTGCTAAGGGGCAG LOC441461: forward CGCACAGGCATACAGATT CTC; reverse CAAGGAAGGGCACTGAAGAG CEP170: forward TCTCCTTGTAAAGCTAAATTC CCCA; reverse AAAAGAGGGAGCAATGCTGA

$G A P D H$ was used as an internal control for normalization. The analysis was performed by comparing CT values (cycle threshold, ie, the cycle where the increase in fluorescence crosses the threshold to be exponential) and the fold change was calculated by using Delta-Delta Ct method.

\section{Results}

Using microarray parameters similar to our prior studies, ${ }^{8,9}$ we found that 14258 genes were differentially expressed between keratoacanthoma and squamous cell carcinoma ( $>2$-fold change (FC): $P<0.05)$. Because of the large number of genes, the data was analyzed with the parameters of $>5 \mathrm{FC}$ $(P<0.01)$ that showed 1449 differentially expressed genes with 908 genes upregulated and 541 genes downregulated.

The most significantly upregulated genes included CDR1, MALAT1, TPM4, CALM1, and TMED2. The most significantly downregulated genes included LOC441461, TYRP1, CEL, INTS6, and WWOX (Table 1).

Hierarchical cluster analysis utilizing the most significant differentially expressed genes revealed that keratoacanthoma and squamous cell carcinoma have distinct genetic signatures (Figure 2). To confirm the reliability of the results from microarray analysis, selected upregulated genes (CDR1, MALAT1, and NCRNA00084) and downregulated genes (LOC441461 and CEP170) were verified by QRT-PCR analyses (Table 2).

The keratoacanthoma samples were also compared with normal skin revealing 15645 differentially expressed ( $>2$ FC: $P<0.05$ ) genes. Because of the large number of genes, the data were analyzed with the parameters of $>5 \mathrm{FC}(P<0.01)$ that showed 2435 differentially expressed genes with 1085 genes upregulated and 1350 genes downregulated. The most significantly upregulated genes included $M A$ LAT1, S100A8, CDR1, TPM4, and CALM1. The most significantly downregulated genes included SCGB2A2, DCD, THRSP, ADIPOQadiponectin, and $A D H 1 B$ (Table 3). The molecular biological pathway analysis comparing keratoacanthoma with normal skin revealed that the most significantly enriched molecular and cellular functions included cellular development, cellular growth and proliferation, cell 
Table 1 Differentially expressed genes distinguishing keratoacanthoma from squamous cell carcinoma

\begin{tabular}{|c|c|c|}
\hline Ref Seq & Symbol/gene & Fold change \\
\hline \multicolumn{3}{|l|}{ Upregulated genes } \\
\hline NM_004065 & CDR1: cerebellar degeneration-related protein 1, $34 \mathrm{kDa}$ & 175.70 \\
\hline NR_002819 & $\begin{array}{l}\text { MALAT1: metastasis-associated lung adenocarcinoma transcript } 1 \\
\text { (nonprotein coding) }\end{array}$ & 156.64 \\
\hline NM_001145160 /// NM_003290 & TPM4: tropomyosin 4 & 86.19 \\
\hline NM_006888 & CALM1: calmodulin 1 (phosphorylase kinase, delta) & 51.38 \\
\hline NM 006815 & TMED2: transmembrane emp24 domain trafficking protein 2 & 49.08 \\
\hline NM_001031827 & BOLA2: BolA homolog 2 (E. coli) & 48.46 \\
\hline NR_000012 & SNORA68: small nucleolar RNA, H/ACA box 68 & 48.01 \\
\hline NM_e15313 & ARHGEF12: Rho guanine nucleotide exchange factor (GEF) 12 & 45.00 \\
\hline NM_001620 /// NM_024060 & AHNAK: AHNAK nucleoprotein & 43.30 \\
\hline NM_012153 & $E H F$ : ets homologous factor & 42.35 \\
\hline \multicolumn{3}{|l|}{ Downregulated genes } \\
\hline XR_041357 /// XR_041358 /// XR_041359 & LOC441461: hypothetical LOC441461 & -28.80 \\
\hline NM 000550 & TYRP1: tyrosinase-related protein 1 & -24.61 \\
\hline NM_001807 & CEL: carboxyl ester lipase (bile salt-stimulated lipase) & -22.68 \\
\hline $\begin{array}{l}\text { NM_001039937 /// NM_001039938 /// } \\
\text { NM_012141 }\end{array}$ & INTS6: integrator complex subunit 6 & -16.63 \\
\hline NM_016373 /// NM_130791 /// NM_130844 & WWOX: WW domain containing oxidoreductase & -15.79 \\
\hline $\begin{array}{l}\text { NM_001042404 /// NM_001042405 /// } \\
\text { NM_014812 }\end{array}$ & CEP170: centrosomal protein $170 \mathrm{kDa}$ & -15.63 \\
\hline $\begin{array}{l}\text { NM_001146274 /// NM_001146283 /// } \\
\text { NM_001146284 /// NM_001146285 /// } \\
\text { NM_001146286 }\end{array}$ & TCF7L2: transcription factor 7-like 2 (T-cell specific, HMG-box) & -13.43 \\
\hline NM_021188 & $Z N F 410$ : zinc-finger protein 410 & -13.42 \\
\hline NM_001047 & $\begin{array}{l}\text { SRD5A1: steroid-5-alpha-reductase, alpha polypeptide } 1 \\
\text { (3-oxo-5 alpha-steroid delta } 4 \text {-de }\end{array}$ & -12.51 \\
\hline NM_001001787 /// NM_001677 & ATP1B1: ATPase, $\mathrm{Na}+/ \mathrm{K}+$ transporting, beta 1 polypeptide & -12.49 \\
\hline
\end{tabular}

death/apoptosis, and cell cycle pathways (Table 4). Examination of the top canonical pathways showed prominent upregulation of clathrin-mediated endocytosis signaling, molecular mechanisms of cancer, and integrin signaling (Table 5).

\section{Discussion}

The DNA microarrays showed that actinic keratoacanthoma and cutaneous squamous cell carcinoma are distinct entities with unique molecular signatures (Figure 2). Our study identified differentially expressed genes and enriched molecular pathways when keratoacanthoma is compared with squamous cell carcinoma and normal skin, laying a strong basis for their separation at the molecular level. Our data showed 1449 differentially expressed genes between keratoacanthoma and squamous cell carcinoma ( $>5$ FC: $P<0.01$ ). The large number of genes differentially expressed suggests that keratoacanthoma is not only a distinct lesion but also is markedly different molecularly from squamous cell carcinoma. Our previous microarray study of the closely related precursor lesion, actinic keratosis ${ }^{8}$ showed only nine genes that were differentially expressed from squamous cell carcinoma ( $>2$ FC: $P<0.05$ ). Additional entities that are molecularly distinct from squamous cell carcinoma include normal $\operatorname{skin}^{8}$ and pseudoepitheliomatous hyperplasia: ${ }^{9}$
382 and 703 differentially expressed genes, respectively ( $>2$ FC: $P<0.05$ ) in our previous microarray studies.

Other studies have similarly shown that keratoacanthoma and squamous cell carcinoma are molecularly distinct. One of the earliest molecular studies utilizing loss of heterozygosity showed multiple differences between keratoacanthoma and squamous cell carcinoma. ${ }^{11,12}$ The frequency of loss of heterozygosity for keratoacanthomas was low with isolated losses at 9p, 9q, and 10q. These results were in contrast to squamous cell carcinoma where loss of heterozygosity was common on chromosome arms 3p, 9p, 9q, 13q, 17p, and 17q. ${ }^{11,12}$ More recent array CGH studies showed significant differences between keratoacanthoma and squamous cell carcinoma in the distribution of numbers of aberrant clones and recurrent aberrations between them. ${ }^{13,14} \mathrm{Li}$ et al14 showed recurrent aberrations in keratoacanthomas on chromosomes 17, 19, 20, and $\mathrm{X}$ in about a third of cases. Recurring aberrations in squamous cell carcinomas were found in $40 \%$ of squamous cell carcinomas on chromosomes $7,8,10,13,17$, and X, with losses on certain regions of $17 p$ and $17 q$ recurring in $50 \%$ of samples. Also, a recent FISH studied showed that EGFR and MYC gene copy number aberrations were more common in squamous cell carcinoma than keratoacanthoma. ${ }^{15}$

There has been much debate as to whether keratoacanthoma is a reactive/hyperplastic or neoplastic 


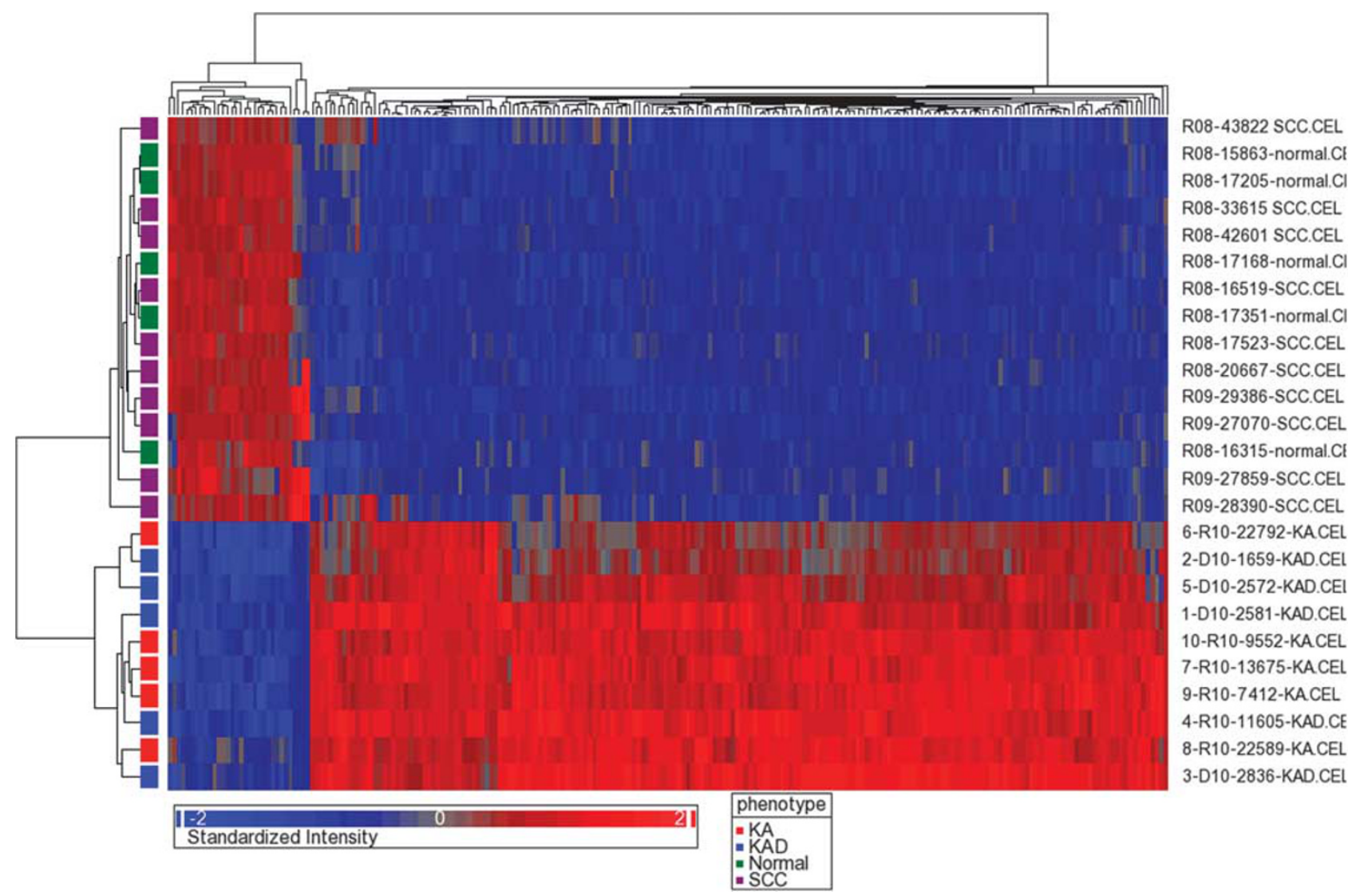

Figure 2 Hierarchical cluster analysis using the most significant differentially expressed genes (y-axis) revealed that keratoacanthoma and squamous cell carcinoma demonstrated distinct genetic signatures. Downregulated genes are in blue and upregulated genes are in red (at 10-fold FDR001-224 genes cluster).

Table 2 QRT-PCR confirmation of the microarray data

\begin{tabular}{lc}
\hline Gene & Average fold change (KA vs. SCC) \\
\hline CDR1 & 2.416 (upregulated) \\
MALAT1 & 2.557 (upregulated) \\
NCRNA00084 & 2.220 (upregulated) \\
LOC441461 & 0.156 (downregulated) \\
CEP170 & 0.290 (downregulated) \\
\hline
\end{tabular}

lesion. In order to gain insight into the pathogenesis of keratoacanthoma, we focused on the altered molecular biologic functions and canonical pathways in comparing keratoacanthoma with normal skin, and the results suggest that keratoacanthoma is neoplastic. Two of the three top canonical pathways: molecular mechanisms of cancer and integrin signaling are well known to be involved in neoplasia. ${ }^{8}$ We have previously reviewed the integrin signaling pathway and its purported role in squamous cell carcinoma carcinogenesis. ${ }^{8}$ Four of the five most significant molecular biological pathways deregulated in keratoacanthoma are related to tumorigenesis and include cellular development, cellular growth and differentiation, cell cycle, and cell movement. In additional support of keratoacanthoma as a neoplastic lesion, the previously discussed molecular studies showed molecular aberrations including loss of heterozygosity and copy number aberrations by CGH that would be expected in a neoplastic lesion. ${ }^{11-14}$ These molecular findings would be unusual in a reactive or hyperplastic process.

Some of the most upregulated genes in comparing keratoacanthoma with normal skin are implicated in neoplasia and include MALAT-1, S100A8, and EHF $(\mathrm{FCs}=216.93,156.65$, and 69.28). MALAT-1 is a long noncoding RNA that is thought to induce tumor migration and growth possibly through the modulation of caspase-3, -8, Bax, Bcl-2, and Bcl-xL. ${ }^{16}$ Its overexpression has been shown to be significantly associated with metastasis and poor prognosis in colorectal, nonsmall cell lung, prostate, pancreas, and cervical carcinomas. ${ }^{16-18}$ EHF is part of the ETS family of transcriptions factors that have been shown to participate in a variety of cellular functions including development, differentiation, proliferation, apoptosis, migration, tissue remodeling, invasion, and angiogenesis. ${ }^{19}$ Fusions of ETS genes with other targets have been described in Ewing's sarcoma, chronic myelomonocytic leukemia, 
Table 3 Differentially expressed genes distinguishing keratoacanthoma from normal skin

\begin{tabular}{|c|c|c|}
\hline Ref Seq & Symbol/Gene & Fold Change \\
\hline \multicolumn{3}{|l|}{ Upregulated genes } \\
\hline NR_002819 & $\begin{array}{l}\text { MALAT1: metastasis-associated lung adenocarcinoma transcript } 1 \\
\text { (nonprotein coding) }\end{array}$ & 216.93 \\
\hline NM_002964 & S100A8: S100 calcium-binding protein A8 & 156.65 \\
\hline NM_004065 & CDR1: cerebellar degeneration-related protein 1, $34 \mathrm{kDa}$ & 146.93 \\
\hline NM_001145160 /// NM_003290 & TPM4: tropomyosin 4 & 109.70 \\
\hline NM 006888 & CALM1: calmodulin 1 (phosphorylase kinase, delta) & 73.16 \\
\hline NM_012153 & $E H F$ : ets homologous factor & 69.28 \\
\hline NM_006815 & TMED2: transmembrane emp24 domain trafficking protein 2 & 66.98 \\
\hline NM_001031827 & BOLA2: BolA homolog 2 (E. coli) & 63.00 \\
\hline NR_000012 & SNORA68: small nucleolar RNA, H/ACA box 68 & 55.07 \\
\hline NM_001114978 /// NM_001114979 /// & TP63: tumor protein p63 & 46.38 \\
\hline $\begin{array}{l}\text { NM_001114980 /// NM_001114981/// } \\
\text { NM_001114982 }\end{array}$ & & \\
\hline \multicolumn{3}{|l|}{ Downregulated genes } \\
\hline NM_002411 & SCGB2A2: secretoglobin, family 2A, member 2 & -132.56 \\
\hline NM_053283 & $D C D$ : dermcidin & -109.61 \\
\hline NM_003251 & THRSP: thyroid hormone responsive (SPOT14 homolog, rat) & -64.04 \\
\hline NM_004797 & ADIPOQadiponectin: C1Q and collagen domain containing & -60.42 \\
\hline NM_000668 & ADH1B: alcohol dehydrogenase 1B (class I), beta polypeptide & -58.21 \\
\hline NM_000517 /// NM_000558 & HBA1 /// HBA2: hemoglobin, alpha 1 /// hemoglobin, alpha 2 & -49.82 \\
\hline NM_006551 & SCGB1D2: secretoglobin, family $1 \mathrm{D}$, member 2 & -48.13 \\
\hline NM_020692 & $\begin{array}{l}\text { GALNTL1: UDP-N-acetyl-alpha-D-galactosamine:polypeptide } \\
\text { N-acetylgalactosaminyltransferase }\end{array}$ & -42.47 \\
\hline NM_173833 & SCARA5: Scavenger receptor class A, member 5 (putative) & -37.36 \\
\hline $\begin{array}{l}\text { NM_001143981 /// NM_001143982 /// } \\
\text { NM } 001143983 / / / \text { NM } 145234\end{array}$ & CHRDL1: chordin-like 1 & -35.17 \\
\hline
\end{tabular}

Table 4 Molecular biological pathway analysis

\begin{tabular}{lcc}
\hline Molecular and cellular functions & P-value & \# Genes \\
\hline Cellular development & 2.91E-15-1.92E-03 & 425 \\
Cellular growth and proliferation & $8.94 \mathrm{E}-13-1.47 \mathrm{E}-03$ & 471 \\
Cell death & $4.90 \mathrm{E}-10-1.79 \mathrm{E}-03$ & 446 \\
Cell cycle & $1.99 \mathrm{E}-09-1.92 \mathrm{E}-03$ & 220 \\
Cellular movement & 8.01E-09-1.88E-03 & 268 \\
\hline
\end{tabular}

Table 5 Top canonical pathways

\begin{tabular}{lc}
\hline Name & P-value \\
\hline Clathrin-mediated endocytosis signaling & $1.10 \mathrm{E}-08$ \\
Molecular mechanisms of cancer & $9.99 \mathrm{E}-07$ \\
Integrin signaling & $2.23 \mathrm{E}-06$ \\
Glucocorticoid receptor signaling & $2.89 \mathrm{E}-06$ \\
Neuregulin signaling & $3.56 \mathrm{E}-06$
\end{tabular}

and prostate carcinoma. ${ }^{20} \mathrm{EHF}$ has been shown to be differentially regulated in prostate, serous ovarian, and breast carcinomas. ${ }^{19-21}$ S100A8 is a calciumbinding protein that was also upregulated in our study comparing squamous cell carcinoma to pseudoepitheliomatous hyperplasia and squamous cell carcinoma to normal skin. ${ }^{8,9}$ It is involved in the regulation of various cellular processes, and its role in tumorigenesis was previously discussed. ${ }^{9}$

Many believe that keratoacanthomas are benign regressing neoplasms. It has been hypothesized that these lesions may be follicle-derived and undergo apoptosis akin to catagen involution of the hair follicle. ${ }^{1,2,5-7}$ Our data supports this hypothesis by showing prominent upregulation of the molecular biological cell death/apoptosis pathway and the canonical clathrin-mediated endocytosis signaling pathway in comparing keratoacanthoma to normal skin (Table 3). Several of the most upregulated differentially expressed genes (CALM1, TP63, YWHAZ, CALR, MMP1, S100A8, and ARHGEF12) are purported to be involved in cell death/apoptosis. The upregulation of these pathways may account for the benign behavior of keratoacanthoma.

Several immunohistochemical studies have also shown that apoptosis has a role in the regression of keratoacanthoma. One study distinguished keratoacanthoma from squamous cell carcinoma by showing that keratoacanthoma expressed markers associated with the initiation (the cytolytic receptor P2X7) and completion phases of (TUNEL) of apoptosis. $^{22}$ Others have shown that keratoacanthoma strongly expresses the proteins bax and bak, which are considered essential for apoptosis execution. ${ }^{23}$ Another study examined keratoacanthomas showed lower expression of the anti-apoptotic protein Bcl-xL that is consistent with a possible role of apoptosis in 
keratoacanthoma regression. ${ }^{24}$ BCL-2 is a proto-oncogene involved in protecting cells from undergoing apoptosis and has been shown to have decreased expression in regressing keratoacanthomas. ${ }^{23,25}$

We hypothesize that prominent enrichment of the clathrin-mediated endocytosis signaling pathway may be because of granzyme-mediated apoptosis. Cytotoxic T cells and natural killer cells utilize lytic effector proteins of perforin and granzymes to eliminate target cells. Cytotoxic T cells have been shown to play an important role in the regression of keratoacanthoma. ${ }^{26}$ Thiery et al. ${ }^{27}$ showed that perforin released by the cytotoxic $\mathrm{T}$ cells activates clathrin- and dynamin-dependent endocytosis to facilitate the internalization of perforin and granzyme by target cells after their release. This is the critical first step initiating target cell apoptosis and may explain the upregulation of clathrin-mediated endocytosis signaling.

The granzyme-mediated apoptosis model also may account for the prominent upregulation of the calcium-binding proteins CALM1 $(\mathrm{FC}=73.16)$. CALM1 encodes calmodulin which is a member of the EF-hand calcium-binding protein family. It encodes a calcium-binding protein that is one of the four subunits of phosphorylase kinase. In the model of granzyme-mediated apoptosis, the action of perforin creates pores in the target cell plasma cell membrane, transiently allowing $\mathrm{Ca}^{2}$ into the cell. The influx of $\mathrm{Ca}^{2}$ leads to a wounded membrane repair response, where lysosomes and endosomes fuse to the plasma membrane to reseal the damaged membrane. This response protects the target cells from necrosis and allows them to undergo the slower, ATP-dependent process of granzyme-mediated apoptosis. ${ }^{27}$ Calmodulin is a calcium-binding protein that may have a role in this response. Increased intracellular calcium in conjunction with calmodulin-associated Fas-mediated apoptosis has been shown in the human B-cell line FMO, Jerkat cells, osteoclasts, and cholangiocarcinoma cells. ${ }^{28}$

The role of S100A8 in tumorigenesis has been previously discussed. However, S100A8 through a complex with S100A9 has also been shown to have a role in tumoral apoptosis in mouse lymphoma and human leukemia cell lines, cervical carcinoma cell lines, and colon carcinoma cells lines. ${ }^{29-31}$ The S100A8/A9 complex has been shown to induce inhibitory effects on the proliferation and invasiveness of tumor cells ${ }^{30}$ through zinc exclusion from target cells and through binding to target cells' surface receptors. ${ }^{31}$

We believe keratoacanthoma is a distinct entity, separate from squamous cell carcinoma. We and others have shown that keratoacanthoma and squamous cell carcinoma have unique molecular signatures. ${ }^{11-14}$ In addition, there is a preponderance of evidence that keratoacanthoma and squamous cell carcinoma have distinct clinicopathological features. ${ }^{1,2,5-7}$ The differentially expressed genes and enriched molecular biological pathways that separate keratoacanthoma from normal skin suggest that keratoacanthoma is a neoplasm that may regress due to upregulation in the cell death/apoptosis pathway. Furthermore, we believe that actinic keratoacanthomas should be treated conservatively and hope that our findings will help prevent radical treatments including wide excision, radiotherapy, and neck dissection for this benign involuting squamous neoplasm.

\section{Acknowledgments}

This paper was presented in part at the 2013 USCAP meeting in Baltimore, MD. We acknowledge Dr Joseph Hillman for his assistance in the selection of keratoacanthoma cases.

\section{Disclosure/conflict of interest}

The authors declare no conflict of interest.

\section{References}

1 Mandrell JC, Santa Cruz D. Keratoacanthoma: hyperplasia, benign neoplasm, or a type of squamous cell carcinoma? Semin Diagn Pathol. 2009;26:150-163.

2 Weedon D. Weedon's Skin Pathology. Elsevier: Edinburgh, UK, 2010;702-708.

3 Hodak E, Jones RE, Ackerman AB. Solitary keratoacanthoma is a squamous-cell carcinoma: three examples with metastases. Am J Dermatopathol. 1993;15: 332-342.

4 Hurt MA. Keratoacanthoma vs. squamous cell carcinoma in contrast with keratoacanthoma is squamous cell carcinoma. J Cutan Pathol. 2004;31:291-292.

5 Godbolt AM, Sullivan JJ, Weedon D. Keratoacanthoma with perineural invasion: a report of 40 cases. Australas J Dermatol. 2001;42:168-171.

6 Savage JA, Maize JC Sr. Keratoacanthoma clinical behavior: a systematic review. Am J Dermatopathol. 2014;36:422-429.

7 Weedon D, Malo J, Brooks D, et al. Keratoacanthoma: is it really a variant of squamous cell carcinoma? ANZ J Surg. 2010;80:129-130.

8 Ra SH, Li X, Binder S. Molecular discrimination of cutaneous squamous cell carcinoma from actinic keratosis and normal skin. Mod Pathol. 2011;24: 963-973.

9 Ra S, Su A, Li X, et al. Molecularly enriched pathways and differentially expressed genes distinguishing cutaneous squamous cell carcinoma from pseudoepitheliomatous hyperplasia. Diagn Mol Pathol. 2013; 22:41-47.

10 Kulkeratoacanthomarni RP, Plaisier S, Ra SH, et al. Genetic profiling of BRAF inhibitor-induced keratoacanthomas reveals no induction of MAP kinase pathway expression. J Invest Dermatol. 2013;133:830-833.

11 Waring AJ, Takeratoacanthomata M, Rehman I, et al. Loss of heterozygosity analysis of keratoacanthoma reveals multiple differences from cutaneous squamous cell carcinoma. Br J Cancer. 1996;73:649-653. 
12 Quinn AG, Sikkink S, Rees JL. Basal cell carcinomas and squamous cell carcinomas of human skin show distinct patterns of chromosome loss. Cancer Res. 1994;54:4756-4759.

13 Clausen OP, Aass HC, Beigi M, et al. Are keratoacanthomas variants of squamous cell carcinomas? A comparison of chromosomal aberrations by comparative genomic hybridization. J Invest Dermatol. 2006; 126:2308-2315.

14 Li J, Wang K, Gao F, et al. Array comparative genomic hybridization of keratoacanthomas and squamous cell carcinomas: different patterns of genetic aberrations suggest two distinct entities. J Invest Dermatol. 2012; 132:2060-2066.

15 Jacobs MS, Persons DL, Fraga GR. EGFR and MYC gene copy number aberrations are more common in squamous cell carcinoma than keratoacanthoma: a FISH study. J Cutan Pathol. 2013;40:447-454.

16 Schmidt LH, Spieker T, Koschmieder S, et al. The long noncoding MALAT-1 RNA indicates a poor prognosis in non-small cell lung cancer and induces migration and tumor growth. J Thorac Oncol. 2011;6:1984-1992.

$17 \mathrm{Xu}$ C, Yang M, Tian J, et al. MALAT-1: a long noncoding RNA and its important 3' end functional motif in colorectal cancer metastasis. Int J Oncol. 2011; 39:169-175.

18 Guo F, Li Y, Liu Y, et al. Inhibition of metastasisassociated lung adenocarcinoma transcript 1 in CaSki human cervical cancer cells suppresses cell proliferation and invasion. Acta Biochim Biophys Sin 2010;42: 224-229.

19 Shaikhibrahim Z, Lindstrot A, Langer B, et al. Differential expression of ETS family members in prostate cancer tissues and androgen-sensitive and insensitive prostate cancer cell lines. Int J Mol Med. 2011;28: 89-93.

$20 \mathrm{He}$ J, Pan Y, Hu J, et al. Profile of Ets gene expression in human breast carcinoma. Cancer Biol Ther. 2007;6: 76-82.

21 Brenne K, Nymoen DA, Hetland TE, et al. Expression of the Ets transcription factor EHF in serous ovarian carcinoma effusions is a marker of poor survival. Hum Pathol. 2012;43:496-505.
22 Slater M, Barden JA. Differentiating keratoacanthoma from squamous cell carcinoma by the use of apoptotic and cell adhesion markers. Histopathology 2005;47: 170-178.

23 Batinac T, Zamolo G, Coklo M, et al. Expression of cell cycle and apoptosis regulatory proteins in keratoacanthoma and squamous cell carcinoma. Pathol Res Pract 8:599-607.

24 Vasiljević N, Andersson K, Bjelkenkrantz K, et al. The Bcl-xL inhibitor of apoptosis is preferentially expressed in cutaneous squamous cell carcinoma compared with that in keratoacanthoma. Int J Cancer. 2009;124:2361-2366.

25 Sleater JP, Beers BB, Stephens CA, et al. Keratoacanthoma: a deficient squamous cell carcinoma? Study of bcl-2 expression. J Cutan Pathol. 1994;21: 514-519.

26 Bayer-Garner IB, Ivan D, Schwartz MR, et al. The immunopathology of regression in benign lichenoid keratosis, keratoacanthoma and halo nevus. Clin Med Res 2004;2:89-97.

27 Thiery J, Keefe D, Saffarian S, et al. Perforin activates clathrin- and dynamin-dependent endocytosis, which is required for plasma membrane repair and delivery of granzyme B for granzyme-mediated apoptosis. Blood 2010;115:1582-1593.

28 Chen Y, Pawar P, Pan G, et al. Calmodulin binding to the Fas-mediated death-inducing signaling complex in cholangiocarcinoma cells. J Cell Biochem. 2008;103: 788-799.

29 Yui S, Mikeratoacanthomami M, Yamazaki M. Induction of apoptotic cell death in mouse lymphoma and human leukemia cell lines by a calcium-binding protein complex, calprotectin, derived from inflammatory peritoneal exudate cells. J Leukoc Biol. 1995; 58:650-658.

30 Qin F, Song Y, Li Z, et al. S100A8/A9 induces apoptosis and inhibits metastasis of CasKi human cervical cancer cells. Pathol Oncol Res. 2010;16:353-360.

31 Ghavami S, Kerkhoff C, Los M, et al. Mechanism of apoptosis induced by S100A8/A9 in colon cancer cell lines: the role of ROS and the effect of metal ions. J Leukoc Biol. 2004;76:169-175. 